\title{
Thermoelectric characterization of flexible micro-thermoelectric generators
}

Cite as: Rev. Sci. Instrum. 88, 015103 (2017); https://doi.org/10.1063/1.4973417

Submitted: 27 September 2016 . Accepted: 14 December 2016 . Published Online: 04 January 2017

D. Beretta, M. Massetti, G. Lanzani, and M. Caironi (D)

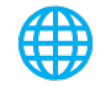

\section{ARTICLES YOU MAY BE INTERESTED IN}

Low-cost flexible thin film thermoelectric generator on zinc based thermoelectric materials Applied Physics Letters 106, 073901 (2015); https://doi.org/10.1063/1.4909531

Interconnect patterns for printed organic thermoelectric devices with large fill factors Journal of Applied Physics 122, 124507 (2017); https://doi.org/10.1063/1.4989589

Flexible and lightweight thermoelectric generators composed of carbon nanotubepolystyrene composites printed on film substrate

Applied Physics Letters 103, 153902 (2013); https://doi.org/10.1063/1.4824648

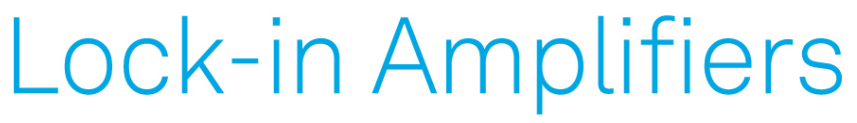

... and more, from DC to $600 \mathrm{MHz}$ Watch 


\title{
Thermoelectric characterization of flexible micro-thermoelectric generators
}

\author{
D. Beretta, ${ }^{1}$ M. Massetti, ${ }^{1,2}$ G. Lanzani, ${ }^{1,2}$ and M. Caironi ${ }^{1}$ \\ ${ }^{1}$ Center for Nano Science and Technology @ PoliMi, Istituto Italiano di Tecnologia, via Pascoli 70/3, \\ 20133 Milano (MI), Italy \\ ${ }^{2}$ Dipartimento di Fisica, Politecnico di Milano, Piazza Leonardo da Vinci 32, 20133 Milano (MI), Italy
}

(Received 27 September 2016; accepted 14 December 2016; published online 4 January 2017)

\begin{abstract}
A new experimental setup for the characterization of flexible micro-thermoelectric generators is reported. The system can measure the power generated and the thermoelectric conversion efficiency of devices under mechanical stresses and deformations, in atmospheric environment and under vacuum, in the temperature interval $293 \mathrm{~K}-423 \mathrm{~K}$, as a function of the load resistance and of the mechanical pressure, with an uncertainty on the temperature difference of $\pm 0.02 \mathrm{~K}$. The system has been tested on commercial rigid devices and on a custom-made, flexible, proof-of-concept, organic-inorganic hybrid generator made of eight thermocouples. Repeatability on the power generated and conversion efficiency within 5\% and 3\%, respectively, was demonstrated, and accuracy of the measurement was granted by minimization of all the potential sources of heat flux losses. Published by AIP Publishing. [http://dx.doi.org/10.1063/1.4973417]
\end{abstract}

\section{INTRODUCTION}

Thermoelectric generation is a solid state technology which provides an effective method to directly convert heat into electricity. It allows for efficiency improvements in a variety of processes affected by thermal losses by partially converting the waste heat into electrical current, and it makes possible powering low energy electronics by exploiting local temperature gradients. A thermoelectric generator (TEG) is made of a number of thermocouples (or $p$ - $n$ junctions) connected electrically in series and forming a thermal parallel, and sandwiched between two electrically insulating layers. When a temperature gradient is established between the junctions, a current is generated according to the thermoelectric properties of the constituent matter. ${ }^{1}$ Thermoelectric generators can operate in the dark and, being made of no moving parts, require almost no maintenance. In the light of their characteristics, they are being investigated for a variety of applications where other technologies are not implementable or are not convenient, these applications spanning from space missions to microelectronics, passing through the industrial and automotive sectors. ${ }^{2}$ Depending on the effectiveness of the thermal coupling with the environment and on the thermal properties of the materials the devices are made of, some applications are well addressed by conventional bulky modules, ${ }^{2}$ while others are better served by microarchitectures, namely, devices characterized by a thickness in the order of few tens of microns. ${ }^{3}$ Sensors and small actuators for the Internet of Things (IoT) belong to the latter case, ${ }^{4}$ where the typical power demanded is below $100 \mu \mathrm{W}$ and Micro-Thermoelectric Generators ( $\mu$ TEGs) operating under small temperature differences can serve as distributed and renewable energy sources for the sustainable charging of batteries. ${ }^{5}$ MTEGs have been designed and realized for the scope according to planar and vertical architectures, ${ }^{3,6-8}$ and flexible thermoelectric generators have been demonstrated, ${ }^{9-13}$ definitely opening the doors to a variety of novel applications in the field of IoT involving flexible electronics, such as selfpowered, wearable, biomedical sensors. ${ }^{14}$ In this framework, organic materials are emerging as potential thermoelectric candidates due to their capability to preserve electronic and mechanical properties even under mechanical stresses and deformations. ${ }^{15-17}$ Among the designs proposed so far, vertical architectures were found to be the best structures for the micro-thermoelectric generation under small temperature differences. In fact, regardless of the power generated and the efficiency of conversion, since the voltage delivered by a TEG is proportional to the number of thermocouples and to the external temperature difference, when the temperature difference is small (2-5 K), a big number of thermocouples is required to generate voltages of practical use, namely, in the interval $100 \mathrm{mV}-5 \mathrm{~V}$. Devices designed with a vertical architecture can accommodate, in principle, a much higher number of thermocouples (up to tens of thousands per $\mathrm{cm}^{2}$ ) with respect to devices based on planar architectures, thus representing, in this context, the most promising architecture. Folding techniques have been recently implemented to recover vertical structures from planar ones, ${ }^{3}$ thus exploiting thin film fabrication methods to realize vertical generators. However, the final device is relatively bulky and its flexibility is limited compared to a vertical device.

Given the interest in micro, flexible, thermoelectric generation under small temperature differences, the thermoelectric characterization of flexible $\mu$ TEGs with vertical architecture, under mechanical stresses and deformations, and small temperature gradients, is becoming fundamental in order to experimentally assess the potentiality of this novel technology in its typical operative conditions. While different systems have been proposed over time for the characterization of bulky and rigid TEGs, each of them exploiting different solutions to measure the incoming heat flux, to apply and stably sustain a mechanical pressure on the device, and to limit radiation heat losses at high temperatures, ${ }^{18-24}$ precise and accurate measurements of micro, flexible devices, under 
mechanical deformation, has not been addressed yet. In this work, a system for the measurement of the power generated and of the conversion efficiency of flexible, microgenerators, as a function of the temperature difference, of the load resistance and of the mechanical pressure, and which can take into account for the effect of devices bending by means of appositely designed device holders, is presented. The system capabilities are assessed on commercial microgenerators from Micropelt and on a custom-made, proof-of-principle flexible microgenerator, fabricated by inkjet printing, under flat and curved configurations.

\section{THEORY OF GENERATORS AND MEASUREMENT METHOD}

To a first approximation, TEGs can be represented as voltage generators controlled in temperature, with internal electrical resistance $R_{T E G}$, and delivering the open circuit voltage $V_{O C}$. The conversion efficiency $\eta$ of a thermoelectric generator is defined as the maximum ratio between the power generated $P_{\text {out }}$ and the incoming heat flux $\dot{Q}_{i n}$, in formula ${ }^{1,23}$

$$
\eta=\frac{P_{\text {out }}}{\dot{Q}_{\text {in }}}
$$

where the power generated is given by the product of the voltage $V_{\text {load }}$ delivered to a load and the current $I$ circulating in the circuit when a load is attached to the generator.

Considering only average values of the thermoelectric coefficients describing the thermoelectric properties of the materials the device is made of, under stationary state hypothesis and fixed external temperatures, $V_{O C}=N \alpha_{p n}\left(T_{h}-T_{c}\right)$, where $N$ is the number of thermocouples, $\alpha_{p n}=\alpha_{p}-\alpha_{n}$ the relative thermopower of the thermocouples, $T_{h}$ and $T_{c}$ the temperature of the hot and the cold side of the thermocouples, respectively. Accordingly, the power generated can be expressed by the following equation: ${ }^{1}$

$$
P_{\text {out }}=\frac{m}{(1+m)^{2}} N Z K_{p n}\left(T_{h}-T_{c}\right)^{2},
$$

where $m=R_{\text {load }} / R_{T E G}$ is the load resistance ratio and $Z$ $=\frac{\alpha_{p n}^{2}}{R_{p n} K_{p n}}$ is the figure of merit of the thermocouple, characterized by the thermoelectric properties of its constituent materials, namely, their relative thermopower $\alpha_{p n}$, their parallel thermal conductance $K_{p n}$ and their electrical series resistance $R_{p n}$. Under the same hypothesis and external conditions, the theoretical maximum efficiency can be approximated by the following equation: ${ }^{1}$

$$
\eta_{\max }=\frac{T_{h}-T_{c}}{T_{h}} \frac{\frac{m}{(1+m)}}{\frac{(1+m)}{T_{h}} \frac{1}{Z}-\frac{T_{h}-T_{c}}{2 T_{h}} \frac{1}{(1+m)}+1},
$$

where the first term, the Carnot's cycle efficiency, represents the upper limit to the efficiency of TEGs, which can be reformulated as $\eta_{\max }=\eta_{\text {Carnot }} \times \eta_{T E, \max }$. Therefore, the higher the temperature difference established between the two ends of the thermocouples for a given heat flux, the higher the power generated and the efficiency of conversion.

Optimization of the efficiency and of the power output requires a different choice of $m$. In particular, while the maximum output power is achieved for $m=1$, maximum efficiency is achieved for $m=\left(1+Z \frac{T_{h}+T_{c}}{2}\right)^{\frac{1}{2}}$. This is a consequence of the non-linear relation between the temperature difference established across the two ends of the thermocouples and the current circulating in the circuit due to the Peltier and Joule effects. Hence the importance of measuring the thermoelectric performances of TEGs as functions of the load resistance.

The theoretical maximum efficiency $\eta_{\max }$ can hardly be obtained in real devices because of electrical and thermal contact resistances, and because of the thermal resistances of the substrates. The electrical contact resistance and the thermal resistance of the substrates are due to materials choice and fabrication methods, and thus can be considered intrinsic properties of the device, which are not influenced by the thermoelectric characterization method. Instead, the thermal contact resistance is a consequence of the thermal coupling with the environment, which, besides varying from application to application as a function of the coupling strategy, strongly depends on the condition under which measurements are taken. ${ }^{25}$

In practice, the efficiency of a thermoelectric generator is given by the maximum ratio between the power generated and the incoming heat flux, for a given load resistance. This is obtained, for a given heat flux, maximizing the temperature difference established across the device by reducing all the potential sources of thermal resistance between the substrates and the heat source and sink. This can be achieved by exerting mechanical pressure between the device and the device holder, using when possible a suitable thermal conductive paste in between, not exceeding the maximum weight tolerable by the device. ${ }^{20,26}$ Hence, the knowledge of the relation between the exerted mechanical pressure, the temperature difference between the device substrates, and the performances of the thermoelectric generator is fundamental to the purpose of the characterization. Accurate measurement of the temperature difference between the substrates is achieved by minimizing the temperature drop along the device holder, generally obtained by using highly thermally conductive materials (such as copper) and by probing the temperatures very close to the device. It might happen, as is for micro- and flexible generators, that the maximum weight applicable to the device is smaller than the one needed to smooth out the thermal contact resistance. In these circumstances, the maximum efficiency is limited, and thus determined, by the mechanical properties of the device, and the residual dependence of the efficiency on the pressure applied provides an indication of how far the measurement is from a perfect thermal coupling condition.

Given Ohm's relation, the power generated by a TEG can be practically measured by indistinctly measuring the couples $V_{\text {load }}$ and $I, V_{\text {load }}$ and $R_{\text {load }}$, or $I$ and $R_{\text {load }}$. On the other hand, in order to calculate the efficiency of conversion, according to Equation (1), the heat flux absorbed by the device must be estimated. This can be directly measured by means of a heat flux meter, or indirectly calculated by means of the Fourier heat law, measuring the temperature profile of a columnar shaped material, the thermal properties of which are well known, or 
by calculating the heat dissipated due to the Joule effect by a heater, provided the heat flux is accurately directed in a given direction and thus collected by the device only. Since the estimation of the heat flux is affected by convection, radiation, and heat conduction throughout cables, all these sources of error must be accurately considered at the design level.

In the system here proposed, the power is calculated by measuring the couple $V_{\text {load }}$ and $R_{\text {load }}$ and taking the ratio

$$
P_{\text {out }}=\frac{V_{\text {load }}^{2}}{R_{\text {load }}},
$$

while the incoming heat flux entering the calculation of the efficiency is directly measured by means of a heat flux meter, such that Equation (1), re-casted as a function of measurable quantities, reads

$$
\eta=\frac{V_{\text {load }}^{2}}{R_{\text {load }} \dot{Q}_{\text {in }}} .
$$

The error committed on the measurement of the power generated is given, at first order, by taking the square root of the summation over the first terms squared of the Taylor expansion of Equation (4), namely,

$$
\delta P_{\text {out }}=\left[\left(\frac{2 V_{\text {load }}}{R_{\text {load }}} \delta V_{\text {load }}\right)^{2}+\left(\frac{V_{\text {load }}^{2}}{R_{\text {load }}^{2}} \delta R_{\text {load }}\right)^{2}\right]^{\frac{1}{2}},
$$

where the $\delta$-quantities represent the uncertainty of the relative variables. $\delta V_{\text {load }}$ can be determined as the standard deviation over $n$ acquisitions, while $\delta R_{\text {load }}$ is related to the uncertainty of the resistance chosen a priori. Similarly, the error committed on the measurement of the efficiency at first order is given by

$$
\begin{aligned}
\delta \eta= & {\left[\left(\frac{2 V_{\text {load }}}{R_{\text {load }} \dot{Q}_{\text {in }}} \delta V_{\text {load }}\right)^{2}+\left(\frac{V_{\text {load }}^{2}}{R_{\text {load }}^{2} \dot{Q}_{\text {in }}} \delta R_{\text {load }}\right)^{2}\right.} \\
& \left.+\left(\frac{V_{\text {load }}^{2}}{R_{\text {load }} \dot{Q}_{\text {in }}^{2}} \delta \dot{Q}_{\text {in }}\right)^{2}\right]^{\frac{1}{1}}
\end{aligned}
$$

where $\delta \dot{Q}_{i n}$ can be experimentally measured by averaging over $n$ acquisitions of the heat flux meter.

\section{SYSTEM DESCRIPTION}

The system here proposed was designed and realized in order to measure the power generated and the efficiency of thermoelectric conversion of a rigid and flexible thermoelectric generator in the temperature interval 293-423 K, both in atmospheric environment and under vacuum down to $10^{-5} \mathrm{mbar}$, as a function of the temperature difference and of the mechanical pressure exerted on the generator. The temperature interval was chosen according to the temperature range tolerated by common materials implemented in the fabrication of flexible devices, such as plastics. A photograph and the technical schematic of the system are shown in Figures 1(a) and 1(b), respectively. It is made of four stainless steel stages, two of which are held fixed, while the others can slide vertically, the handling system being provided by four steel bars and a set of ball bearings. Heating/cooling subsystems are mounted on the two sliding stages, and on such subsystems two replaceable copper towers, responsible

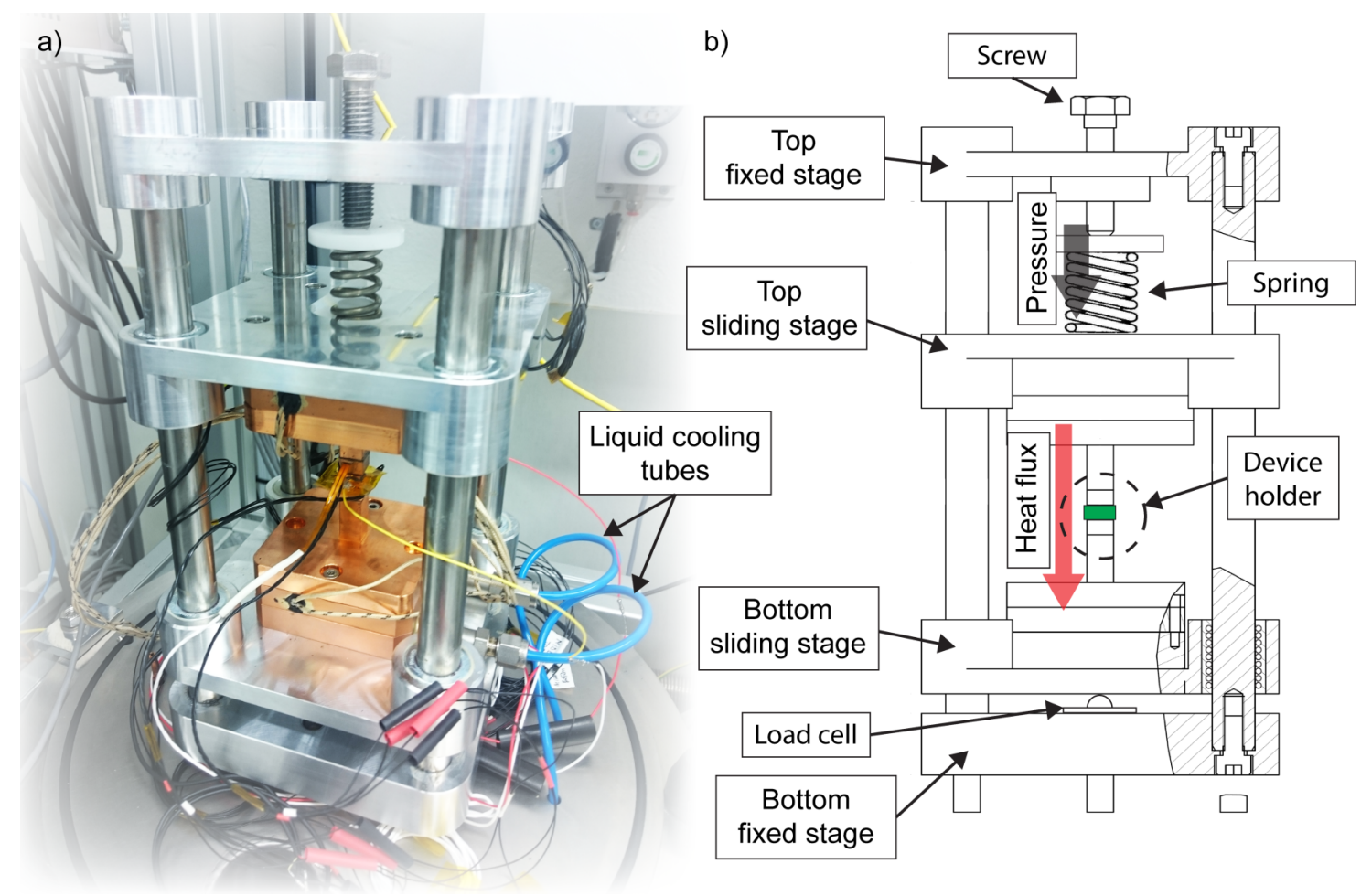

FIG. 1. Photograph (a) and schematic (b) of the system. The device holder is pressed between two copper towers which are symmetrically installed on heating/cooling subsystems. A spring mounted between the top stages allows to exert a certain mechanical pressure on the device, which is measured by a load cell installed between the two bottom stages. 
for steering the heat flux throughout the device, are installed. The device holder is mounted between the two copper towers. An interchangeable spring, characterized by a high elastic coefficient, is mounted between the two top stages and is kept on axes by a spring holder made of two symmetrical plates of Teflon. The top fixed stage is provided by a M12 screw. Rotating the screw, its head pushes the top Teflon plate, which compresses the spring and indirectly transmit mechanical pressure to the device. The pressure is measured by a load cell installed between the two bottom stages, which is capable of measuring the force exerted in the range $1 \mathrm{~kg}-45 \mathrm{~kg}$.

Heating of the copper towers is provided by two cartridge heaters (100 W Rotfil) installed in two cavities opened in the copper tower bases and glued with a thermally conductive epoxy (Aremco-Bond 2315), while cooling is provided by a water based circuit $(200 \mathrm{ml} / \mathrm{min}$ of water at the temperature of about $283 \mathrm{~K}$ ), brazed into the copper tower bases. Heating is controlled by two PID (proportional-integral-derivative) units (Omron E5CN-H) which read as an input the temperature signal coming from two 4-wires PT100 (Tersid) installed in the device holder and allow the temperature stabilization of the device holder with precision up to $\pm 0.01 \mathrm{~K}$.

The incoming heat flux, required to extract the efficiency of conversion according to Equation (5), is measured by a replaceable high resolution heat flux meter (greenTEG gSKIN@) installed between the upper copper tower and the upper side of the device holder. The sensor can resolve heat fluxes down to $10 \mu \mathrm{W} \mathrm{cm}{ }^{-2}$ and is thermally anchored by means of a thermally conductive paste (Arctic Silverß 5).

The device holder, mounted and pressed between the two copper towers, is made of two small interchangeable copper blocks which can be realized on demand in order to accommodate devices of different dimensions and geometries, with a maximum height of $3 \mathrm{~cm}$ and maximum side length of $4 \mathrm{~cm}$, and/or under mechanical stresses and deformations. A schematic of the device holder is shown in Figure 2.

The device is sandwiched between the copper blocks. Each copper block is provided by a 4-wire thin film PT100 (Tersid) temperature sensor, installed in a dedicated hole and thermally anchored with a thermally conductive epoxy (Aremco-Bond 2315), used as input signals for the PIDs and to measure with high precision and high accuracy the temperature at both sides of the device. The thermal contact between the device holder and both the copper towers and the device is provided by a thermal conductive paste (Arctic Silver 5). The installation method chosen permits to easily substitute the device holder, such that device holders with a curved surface can be implemented in order to perform measurements on flexible devices and determine the effect of the radius of curvature on the efficiency of conversion and on the power output.

Due to the position of the heat flux sensor, radiation losses affecting the measurement of the efficiency are limited to the lateral surface of the device holder, which turns out to be in the order of $10^{-4} \mathrm{~m}^{2}$. From the Stefan-Boltzmann law and considering the emissivity of oxidized copper equal to 0.78 , the power radiated by the device holder amounts to less than $1 \mathrm{~mW}$ at $473 \mathrm{~K}$. Since the typical heat flux imposed externally is

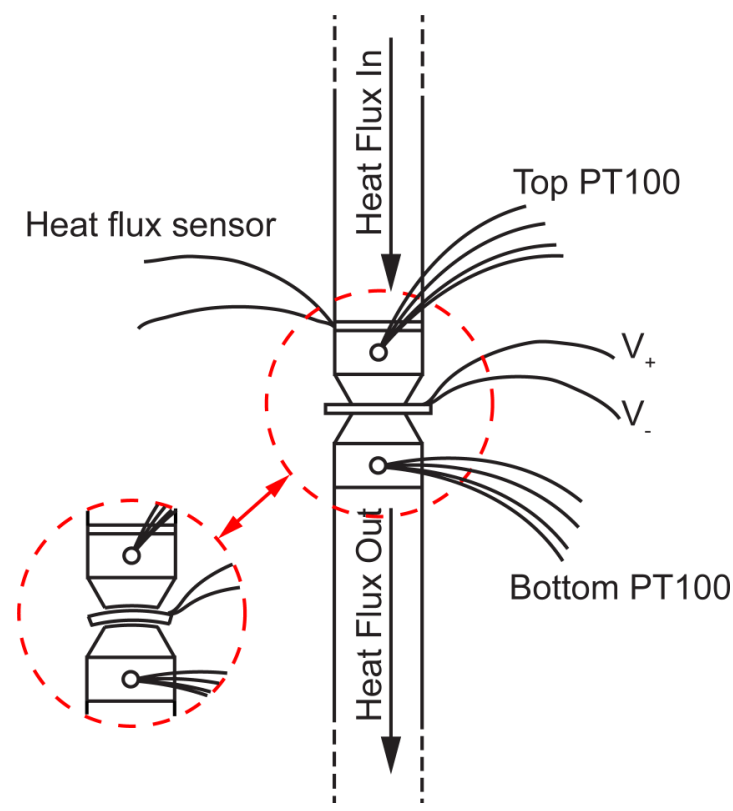

FIG. 2. Schematic of the replaceable device holder sandwiched between the two copper towers. Positions of the heat flux sensor, the PT100s, and the device under test are marked.

higher than few hundreds of $\mathrm{mW}$, the power radiated represents a negligible quantity which does not affect the measurement of the efficiency. For this reason, the system is not equipped with a thermal shield. Under vacuum, the measurement of the heat flux may thus be affected only by the heat losses due to heat conduction throughout the cables of the PT100 sensor installed on the top copper block of the device holder. Given the diameter and the length of these copper cables, $100 \mu \mathrm{m}$ and $2 \mathrm{~m}$, respectively, the outgoing heat flux through the cables is in the order of few hundreds of $\mu \mathrm{W}$ under a temperature difference of $100 \mathrm{~K}$ and is therefore negligible. In the light of these considerations, the measurement of the efficiency of conversion under vacuum can be considered affected only by the uncertainty of the heat flux sensor and by the uncertainty on the estimation of the power generated. On the contrary, measurements in atmospheric environment are necessarily affected by convection throughout the lateral surface of the device holder, resulting in an inaccurate estimation of the incoming heat flux and thus of the efficiency of conversion, as it will be shown experimentally in Section IV.

All the cables carrying signals are taken out of the vacuum chamber through a 25 sub-miniature D multipin feedthrough (Allectra), and the cables carrying the voltage generated by the device are soldered to a rotary switcher, to which a number of different load resistances appropriately chosen are connected.

The heat flux and the TEG voltages are acquired in real time by a nanovoltmeter (Keithley 2182A) in cascade to a multiplexer (Keithley 7001) mounting a nanovolt scanner card (Keithley 7168), while the temperature signals are acquired in real time by reading the PIDs' temperature registers following an RS-485 communication protocol, and a LabView based GUI is used for real time monitoring of every signals. A schematic of the connections between sensors and electronics is shown in Figure 3. A Matlab code finally allows elaborating 


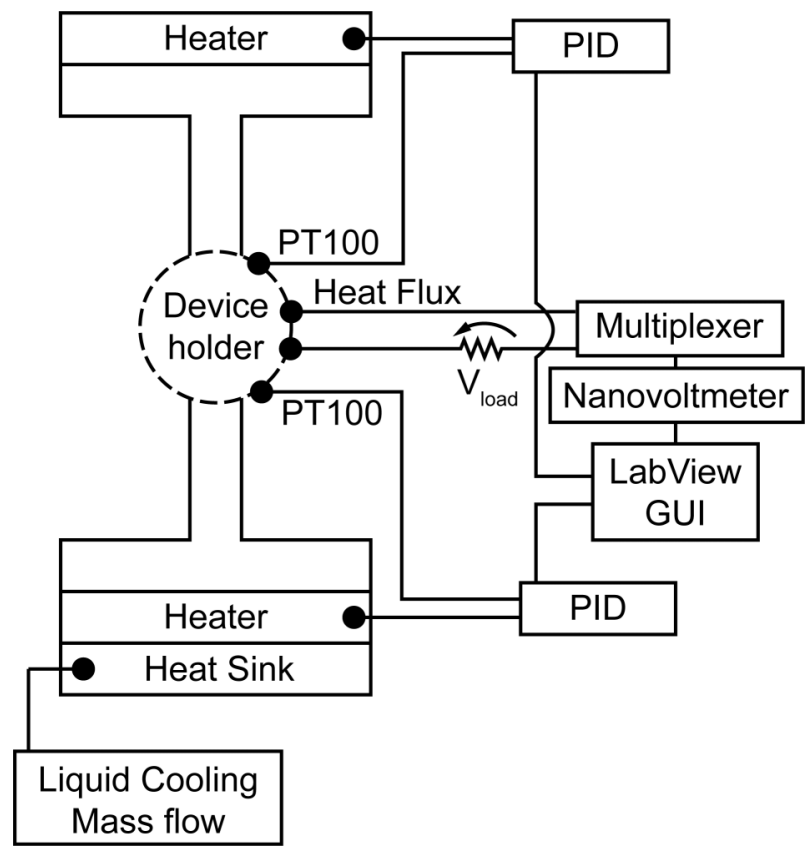

FIG. 3. Schematic of the system. The signal coming from the heat flux and the load voltage is acquired by a nanovoltmeter in cascade to a multiplexer. The temperature signal of the two PT100s installed in the device holder is used as an input for the PIDs. A LabView based GUI is used for real-time monitoring.

in one shot the measurements taken as a function of the load resistance and of the temperature difference.

\section{SYSTEM VALIDATION}

In order to validate the capability of the system to measure rigid and flexible $\mu$-TEGs, three commercial rigid microgenerators from Micropelt (MPG-D655) and an appositely designed and fabricated, proof-of-principle, flexible, microorganicinorganic hybrid generator were tested. Repeatability of the measurements was assessed by loading and unloading a single rigid device a number of times, and the effect of the mechanical pressure was studied by performing measurements on a rigid device, at load matching, under different weights. Results of the measurements are discussed in Subsections IV A-IV D.

\section{A. Characterization of rigid devices}

The thermoelectric characterization of the three generators from Micropelt (namely, TGP-651-1, -2, and -3) was done in vacuum, using a device holder with a flat square section of $1 \mathrm{~cm}^{2}$, under a mechanical weight of $2 \mathrm{~kg}$, with the cold side held fixed at $298 \mathrm{~K}$ and the temperature difference spanning the interval $5-75 \mathrm{~K}$. The resistance of the generators was found to span the interval $210-260 \Omega$ in the temperature range 300-370 K (see Figure 4). In particular, while TGP-651-2 and -3 were found to be electrically similar, TGP-651-1 showed a slightly higher value of resistance.

The thermoelectric characteristics of TGP-651-1, namely, the I-V curves, the power generated, and the efficiency of conversion are shown in Figures 5(a)-5(c), respectively. The

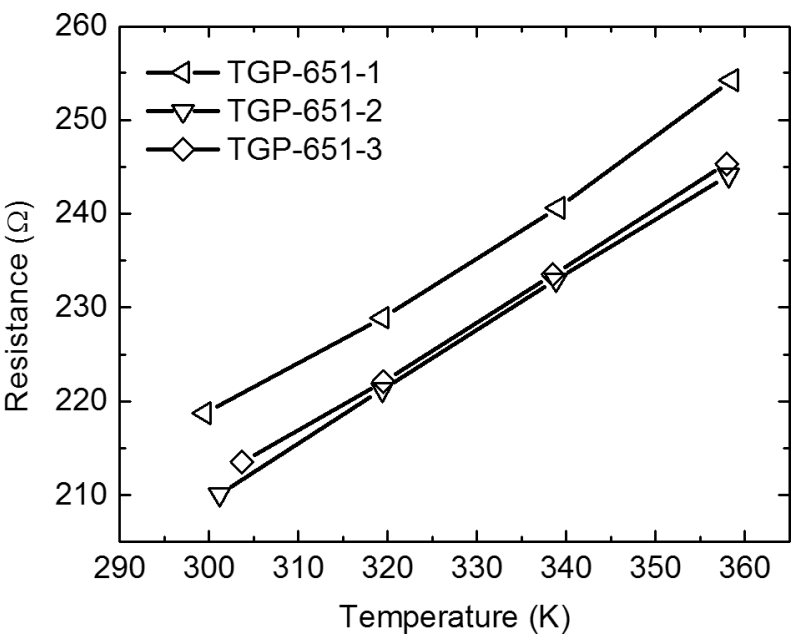

FIG. 4. Electrical resistance versus temperature of the generators from $\mathrm{Mi}$ cropelt. TGP-651-1 shows a slightly higher resistance with respect to the other two.

standard deviation of the data reported, not shown, was calculated according to Equations (6) and (7) and was found to fall within $0.1 \%$ of the average values. The generators show an ohmic behavior in the whole temperature range investigated, and the power and the efficiency curves follow the typical trend expected from the theory, with a maximum at the corresponding load matching. TGP-651-1 is observed to show slightly different performances with respect to the other two devices, in agreement with the slightly different resistance values measured in the whole temperature range of investigation. Comparisons between power at load matching and open circuit voltage measured with the system here presented (black) and data from manufacturer (red) are shown in Figures 5(d) and 5(e), respectively. In detail, manufacturer data are denoted with SK422-33-SA, -50-SA, and -90-SA, the code referring to the type of heat exchangers (Fischer Elektronik) mounted at the cold side, characterized by a length of $33 \mathrm{~mm}, 50 \mathrm{~mm}$, and $90 \mathrm{~mm}$, respectively. The measured functional trends with temperature are identical. Measurements from this work and data from manufacturer differ only slightly and they are found to be in agreement with what expected considering the different mechanisms of thermal coupling with the environment employed for the characterizations. In fact, while the manufacturer data refer to a condition in which the heating is provided by a direct mechanical contact between the device and the heat source, and the cooling by a heat exchanger under natural convection installed on top of the device, the system here presented makes use of the direct mechanical contact between the generator and both the heating and the cooling sources. This generally results in smaller thermal resistances between the device and the heat source/sink, ultimately leading to a higher effective temperature difference falling across the device, and thus to a higher power output and efficiency of thermoelectric conversion. The capability of the system to measure rigid devices is thus assessed. The efficiency, under condition of power load matching, is shown in Figure 5(f). Despite data on efficiency were not available from the manufacturer, the values achieved are reasonable for state-of-the-art microgenerators. 
a)

b)
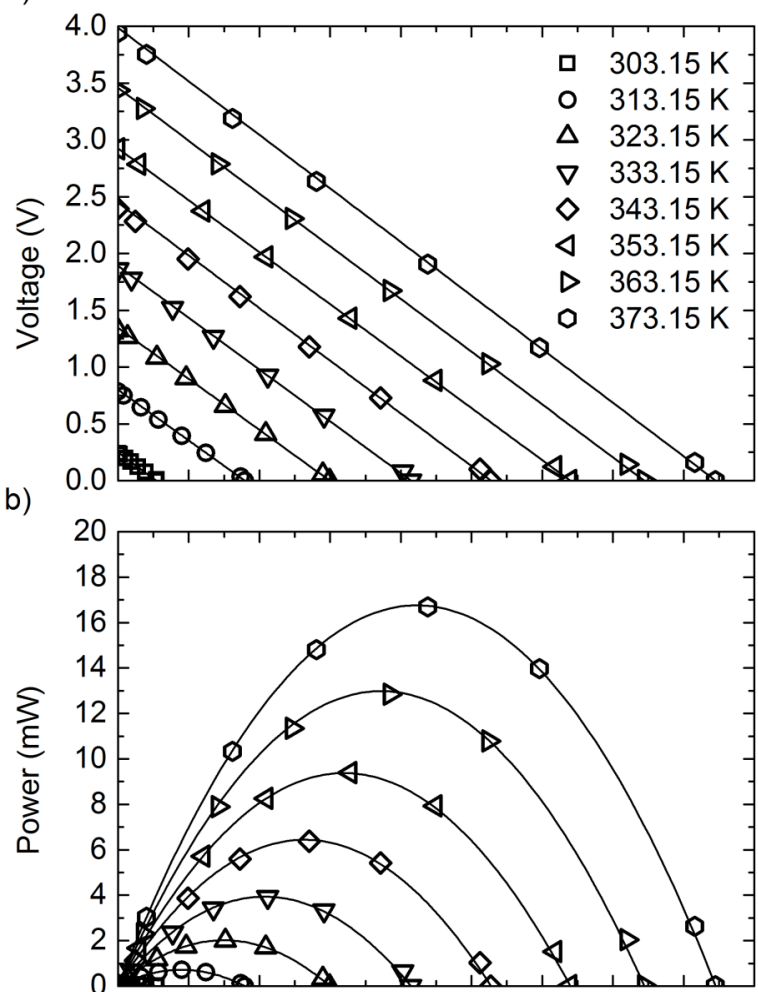

c)

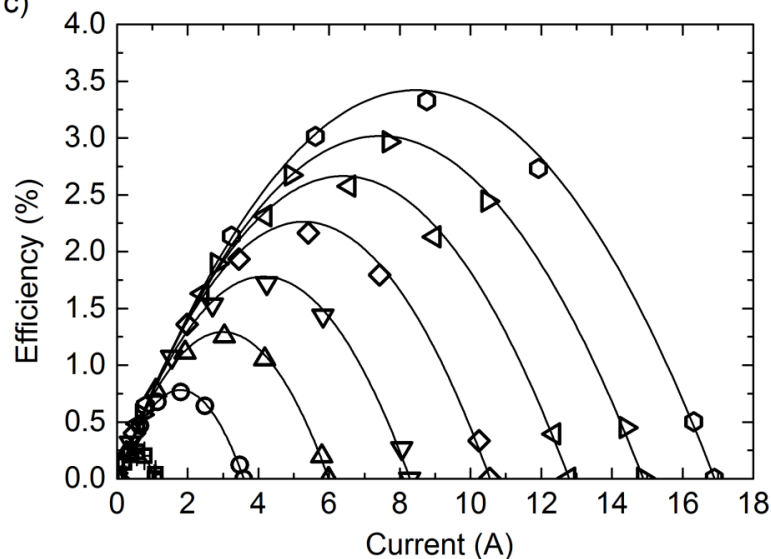

d)

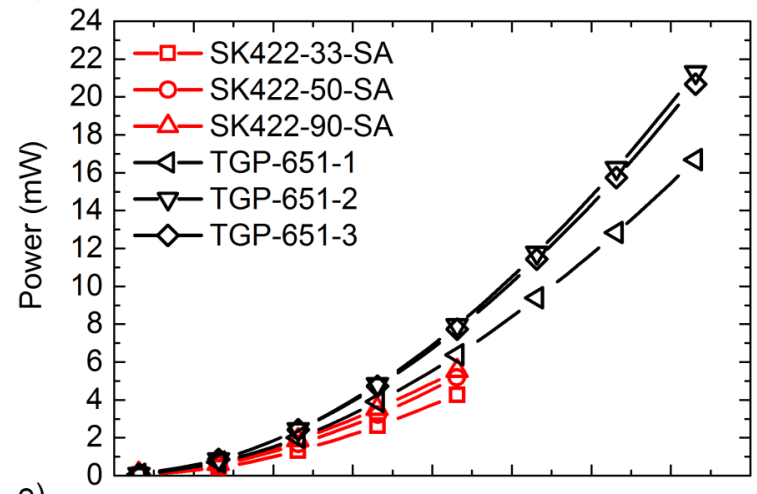

e)

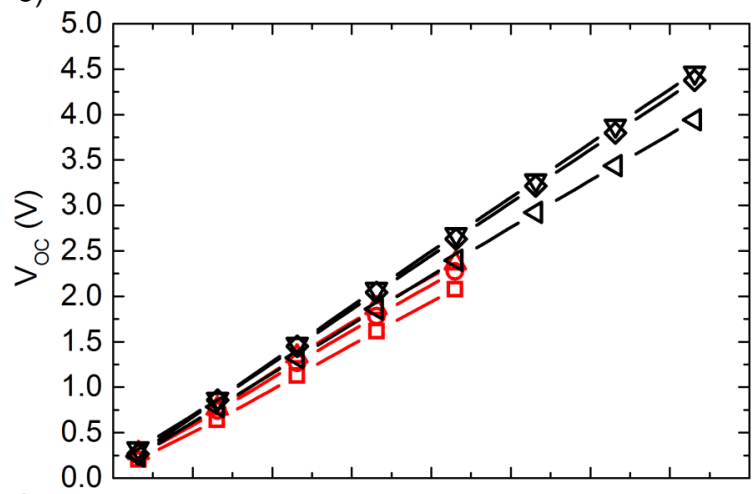

f)

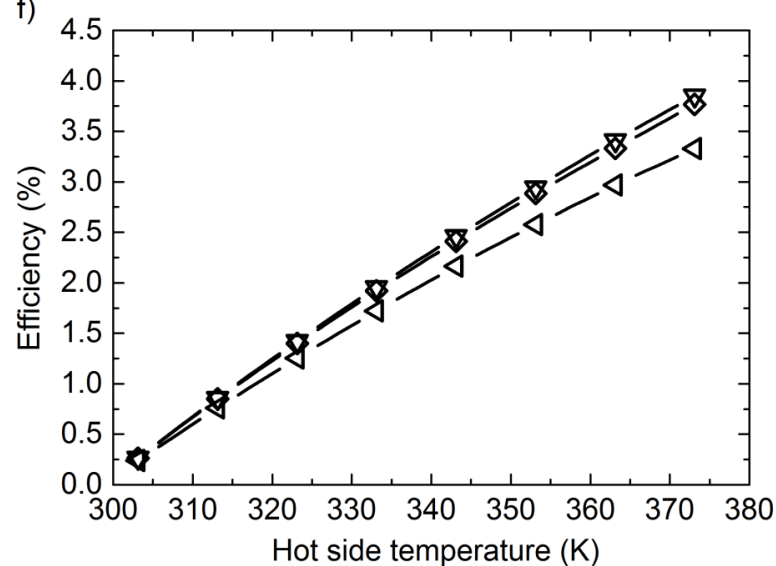

FIG. 5. I-V curves (a), power generated versus current (b), and efficiency of thermoelectric conversion (c) of TGP-651-1. The generator shows the ohmic behavior in the whole temperature interval of measurements, and power and efficiency reveal the typical curve expected from the theory, with a maximum at the corresponding load matching condition. Comparison between power at load matching (d) and open circuit voltage (e) validate the capability of the system. Efficiency of conversion at power load matching is shown in panel (f). Small differences between measurements and data from manufacturer are in accord with the different method of thermal coupling with the environment employed.

\section{B. Characterization of flexible devices}

In order to assess the capability to measure micro, flexible devices, a flexible thermoelectric generator made of 8 thermocouples of PEDOT:PSS/Ag was appositely designed and realized. The device measured is a portion of the larger structure shown in Figure 6 (details of which will be reported elsewhere), which comprises 312 thermocouples per $\mathrm{cm}^{2}$ and has an overall thickness of approximately $75 \mu \mathrm{m}$. The device is realized on a $25 \mu \mathrm{m}$ thick polyimide substrate (Kapton DuPont) and it is made of $25 \mu \mathrm{m}$ thick thermocouples deposited by inkjet printing (Fujifilm Dimatix DMP-2831, $10 \mathrm{pL}$ droplet volume) in cavities micro-fabricated in a photoresist film (SU-8 Microchem) appositely exposed and developed. $200 \mathrm{~nm}$ thick gold metallic interconnections are realized by thermal PVD (Physical Vapor Deposition) (Braun MB-ProVap-3) and the top insulating layer is provided by a $25 \mu \mathrm{m}$ thick hardened film of photoresist (SU-8 Microchem).

The thermoelectric characterization was done in vacuum, under a mechanical weight of $2 \mathrm{~kg}$, with the cold side kept at $298 \mathrm{~K}$ and for two different temperature differences, namely, $5 \mathrm{~K}$ and $15 \mathrm{~K}$. The device was first measured in a flat configuration, and then under a radius of curvature of $10 \mathrm{~mm}$ by means of an appositely designed device holder, having a section of approximately $1 \mathrm{~cm}^{2}$. Load matching at room temperature was achieved for $R_{\text {Load }}$ approximately equal to $3.3 \mathrm{k} \Omega$ in the flat configuration and $12 \mathrm{k} \Omega$ in the curved configuration. The thermoelectric characteristics 


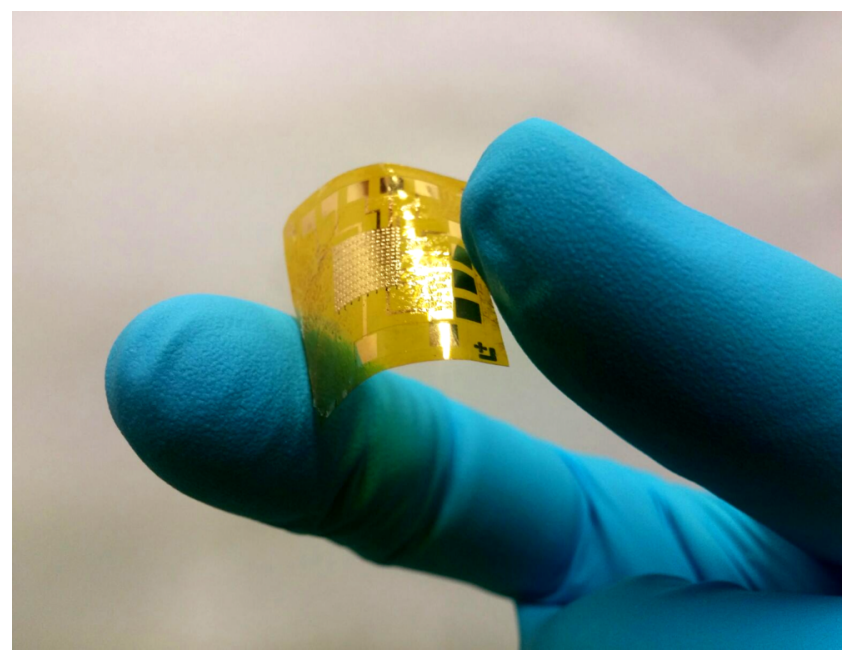

FIG. 6. Photograph of the flexible thermoelectric device designed and fabricated appositely for the testing of the system. For the purpose of the measurement, only 8 thermocouples were connected and studied.

of the device are shown in Figure 7, where data from flat (black) and curved (red) measurements are superimposed. The device shows the ohmic behavior, in both conditions, in the whole temperature range investigated (panel (a)). The generated power (panel (b)) and the efficiency of conversion (panel (c)), the latter normalized with respect to the effective device section crossed by the heat flux, follow the typical trend observed in conventional inorganic, rigid generators, showing a maximum at the corresponding load matching. Differences are observed among the thermoelectric characteristics of the flat and the curved configurations. A discussion on the origin of those discrepancies, which could be likely ascribed to reduced conductivity and/or structural modification of the thermocouples and their metallic interconnections due to mechanical deformations, is out of the scope of this work. The thermoelectric performances of the generator, despite poor, are in agreement with the typical electrical and thermal properties of the materials implemented ${ }^{27}$ and with the device architecture adopted, made of only eight thermocouples, electrically and thermally non-optimized. ${ }^{25}$ In fact, considering a linearized thermoelectric model, ${ }^{28}$ the effective temperature difference falling across the thermocouples for such architectures is in the order of $0.1 \%$ of the externally applied temperature difference, owing to the use of highly thermally conductive Ag legs, with a section equal to the one of the PEDOT:PSS legs. The latter choice, mainly deriving from the scarcity of stable, printable, and highly electrically conductive organic n-type materials, leads to very small open circuit voltage (tens of $\mu \mathrm{V}$ under temperature differences of tens of $\mathrm{K}$ ) and current, and thus to small power and efficiency of conversion. The system capability to measure flexible, microdevices, under small temperature differences and under mechanical deformation is validated. In addition, the system was shown to be capable of measuring devices generating currents in the order of $\mathrm{nA}$ and to resolve voltages in the order of tens of $\mu \mathrm{V}$, thus allowing to characterize the thermoelectric properties of devices made of a small number of thermocouples, as required in research and/or a)

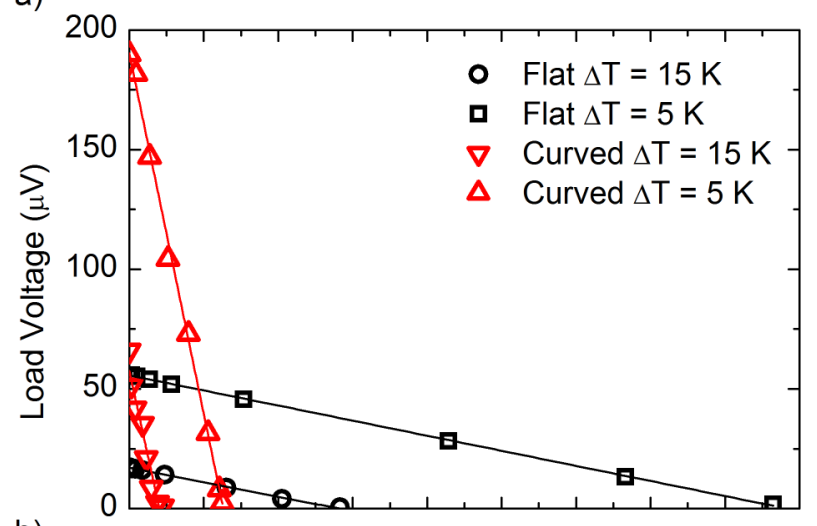

b)
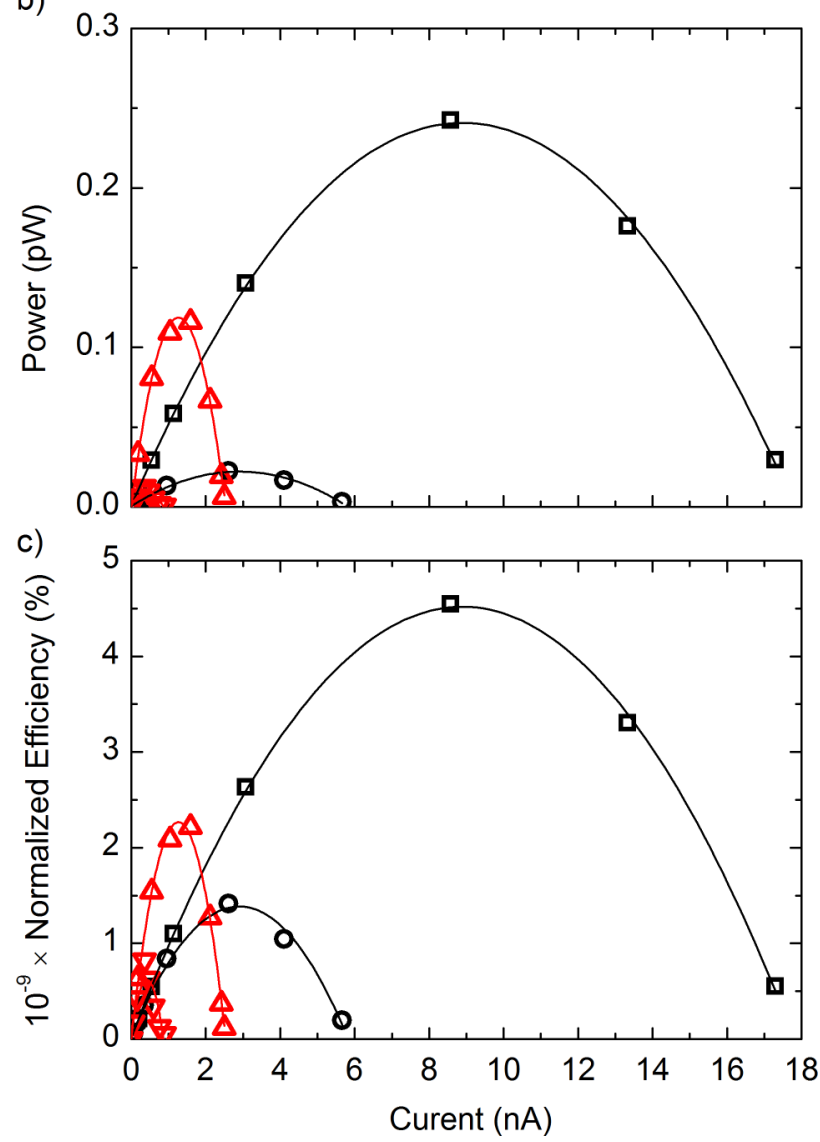

FIG. 7. I-V curves (a), power generated (b), and normalized efficiency (c) of a flexible, organic-inorganic hybrid micro-thermoelectric generator. Measurements from flat (black) and curved (red) configurations are superimposed.

development activities, and/or under very small temperature differences.

\section{Measurement uncertainty}

In order to determine the uncertainty of the measurement due to device loading procedure, TGP-651-3 was loaded and unloaded five times in atmosphere and five times under vacuum, and the generated power and the efficiency of conversion were measured at load matching under a temperature difference of $25 \mathrm{~K}$, the cold side being held at $298 \mathrm{~K}$, and under a mechanical weight of $2 \mathrm{~kg}$ (data shown 


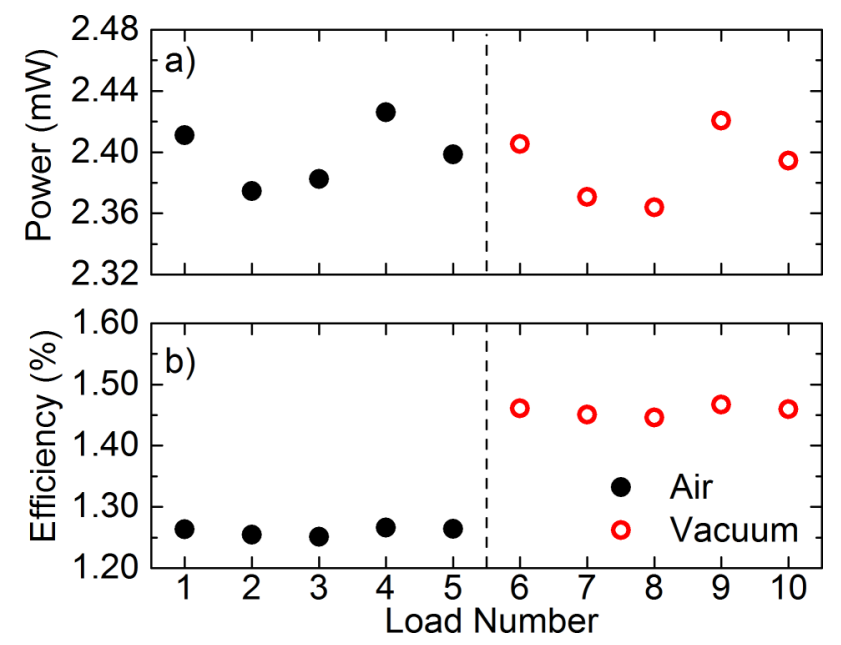

FIG. 8. Power generated at load matching (a) and corresponding efficiency of conversion (b) measured on TGP-651-3 after different loadings, in atmosphere (black) and under vacuum (red), and under a temperature difference of $25 \mathrm{~K}$. Overall power and efficiency variation is below $3.5 \%$ and $2 \%$, respectively.

in Figure 8). The dispersion of data on the generated power and on the efficiency of conversion was found to be less than $3.5 \%$ and $2 \%$, respectively, in both the atmospheric and vacuum environments, confirming the excellent repeatability of the measurements performed with the system proposed in this work. While the power generated in atmosphere and vacuum does not differ among themselves, the efficiency measured in vacuum is higher than the one measured in atmosphere by approximately $15 \%$. This effect is ascribed to the convection phenomena affecting the lateral surfaces of the device holder: in order to sustain a given temperature difference between the two copper blocks of the device holder in atmosphere, the heat flux imposed must compensate for the thermal losses arising from convection and thus must be higher with respect to the case of vacuum insulation. These observations indicate that in order to measure the maximum efficiency of energy conversion with the system here proposed, vacuum environment is necessary.

\section{Mechanical pressure dependency}

In order to determine the effect of the mechanical pressure on the efficiency and on the power generated, TGP-651-3 was measured in atmosphere and under vacuum, under mechanical weights spanning the interval $2-20 \mathrm{~kg}$, the corresponding pressure interval being $0.2-2$ bars. The results of the measurements are shown in Figure 9. Both the power and efficiency at load matching are weak increasing functions of the mechanical pressure, with a relative percentage variation of approximately $5 \%$ and $3 \%$, respectively, for weight spanning the interval $2 \mathrm{~kg}-20 \mathrm{~kg}$. This phenomenon is ascribed to the contact thermal resistance between the device and the device holder, which determines a finite temperature drop, ultimately reducing the effective temperature difference falling across the thermocouples relatively to the temperature difference established between the heat source and the heat sink. Increasing the mechanical pressure, the contact

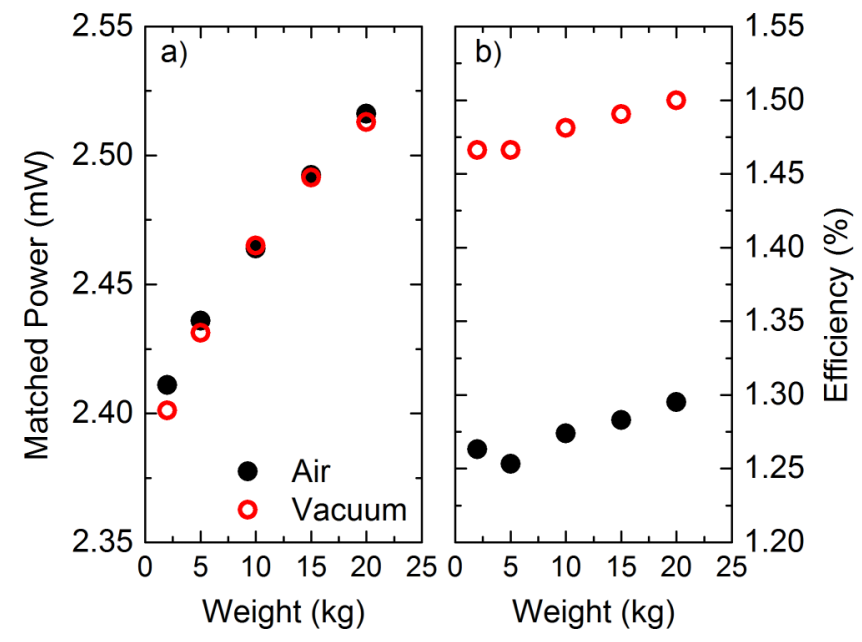

FIG. 9. Power (a) and efficiency (b) at load matching as a function of the mechanical pressure. Both power and efficiency are increasing function of the mechanical pressure. While the power measured under vacuum and in atmosphere are coincident, the efficiency measured under vacuum is found to be higher than the efficiency measured in air. This discrepancy is a direct consequence of convection, which is responsible for the heat exchange throughout the lateral surface of the sample holder when measurements are taken in air, ultimately resulting in higher heat fluxes necessary to sustain a given temperature difference, and thus lower measured efficiency, with respect to the case of measurements under vacuum.

resistance is smoothed out, ${ }^{29}$ the corresponding temperature drop reduced, and the power and the efficiency of conversion tend to their maximum values. In the ideal case, the contact resistance approaches zero as the pressure goes to infinity. However, devices are designed to tolerate a limited interval of weights, and characterization must take into account for such limitations. For instance, the manufacturer states that devices from Micropelt can tolerate a maximum weight of $35 \mathrm{~kg}$ at $373 \mathrm{~K}$. In the particular case of flexible devices, mechanical pressure could also induce mechanical deformations and thus architecture modifications, making the relation between the pressure and contact thermal resistance not straightforward. In this sense, the effect of the mechanical pressure has still to be studied on flexible devices, and the system here presented represents a potential instrument for such investigations. While the power measured in atmosphere and under vacuum at a fixed temperature difference is found to be coincident in the whole interval of mechanical weights, the efficiency measured in atmosphere differs from the efficiency measured under vacuum by approximately $15 \%$ (see Figure 9(b)) in the whole interval of applied weights, confirming the observations on Figure 8(b).

\section{CONCLUSIONS}

A system for the thermoelectric characterization of rigid and flexible, micro-thermoelectric generators was designed and realized. The system is capable to measure the power generated and the efficiency of thermoelectric conversion in atmosphere and under vacuum (down to $10^{-5}$ mbar), as a function of the temperature difference with a precision of $\pm 0.02 \mathrm{~K}$, of the load resistance, and of the mechanical pressure exerted on the device. The mechanical pressure 
exerted on the device, responsible for the thermal contact resistance between the device and the device holder, and thus of the effective temperature difference falling across the generator, was shown to have a small impact on the accuracy of the measurement, the power generated and the efficiency of conversion varying only within $5 \%$ and $3 \%$, respectively, for weight spanning the interval $2 \mathrm{~kg}-20 \mathrm{~kg}$. The system was designed in order to accommodate a replaceable device holder, this allowing for different holders with different shapes in order to study the thermoelectric performances of rigid and flexible devices characterized by different dimensions and/or subjected to certain stresses and deformations. This represents a step forward in flexible devices characterization, where thermoelectric performances of devices under typical operative conditions, such as mechanical stresses and deformations, must be studied in order to assess the potentiality of this novel technology to be practically integrated into flexible electronics. Moreover, since mechanical weights could, in principle, cause architecture deformations, the system allows to investigate these effects on the power generated and efficiency of conversion of flexible devices by tuning the mechanical pressure.

Measurements repeatability was assessed by loading and unloading a single device multiple times, and measuring its performances at load matching. Power and efficiency uncertainties were both found to be below $3.5 \%$ and $2 \%$ in air and under vacuum, respectively, and were ascribed to the loading procedure, the uncertainties determined by signal fluctuations being much smaller $(\approx 0.1 \%$ of the average values). While the power measured in atmosphere and under vacuum was observed to be coincident, the efficiency shows differences on the basis of the environment of characterization. In particular, the efficiency measured in atmosphere was lower than the one measured under vacuum, and the difference was found to be in the order of $15 \%$. This discrepacy is ascribed to convection, which is responsible for heat exchange throughout the lateral surface of the sample holder when measurements are taken in air: in order to sustain a certain temperature difference across the device, losses due to convection must be compensated by applying a bigger heat flux with respect to the case of measurements under vacuum, where convection is absent. This results in a smaller measured efficiency in atmospheric environment. Therefore, in order to estimate the maximum efficiency of thermoelectric conversion, vacuum was demonstrated to be necessary for the system here presented.

The system proposed paves the way towards a systematic investigation and a better understanding of the thermoelectric performances of flexible micro-thermoelectric generators, among the most interesting candidates for the distributed energy supply within the rising IoT field, under the typical mechanical stresses and deformations they are designed for. Such an instrument is thus a powerful support for the design, development, and engineering of future flexible thermoelectric generators.

${ }^{1}$ A. F. Ioffe, Semiconductor thermoelements, and Thermoelectric cooling (Infosearch, ltd., 1957).

${ }^{2}$ L. E. Bell, Science 321, 1457-1461 (2008).

${ }^{3}$ W. Glatz, E. Schwyter, L. Durrer, and C. Hierold, J. Microelectromech. Syst. 18, 763-772 (2009).

${ }^{4}$ S. Greengard, The Internet of Things (The MIT Press, 2015).

${ }^{5}$ D. Briand, E. Yeatman, S. Roundy, O. Brand, G. K. Fedder, C. Hierold, J. G. Korvink, and O. Tabata, Micro Energy Harvesting (John Wiley \& Sons, 2015), Vol. 12.

${ }^{6}$ G. J. Snyder, Electrochem. Soc. Interface 17, 54-56 (2008)

${ }^{7}$ N. Kouma, T. Nishino, and O. Tsuboi, J. Micromech. Microeng. 23, 114005 (2013).

${ }^{8}$ H. Bottner, J. Nurnus, A. Schubert, and F. Volkert, in 2007 26th International Conference on Thermoelectrics (IEEE, 2007), pp. 306-309.

${ }^{9} \mathrm{Y}$. Iwasaki and M. Takeda, in 25th International Conference on Thermoelectrics, 2006 ICT'06 (IEEE, 2006), pp. 562-565.

${ }^{10}$ P. Fan, W. F. Fan, Z. H. Zheng, Y. Zhang, J. T. Luo, G. X. Liang, and D. P. Zhang, J. Mater. Sci.: Mater. Electron. 25, 5060-5065 (2014).

${ }^{11}$ D. Madan, Z. Wang, P. K. Wright, and J. W. Evans, Appl. Energy 156, 587-592 (2015).

${ }^{12}$ Y. Du, K. Cai, S. Chen, H. Wang, S. Z. Shen, R. Donelson, and T. Lin, Sci. Rep. 5, 6411 (2015).

${ }^{13}$ J. A. Lee, A. E. Aliev, J. S. Bykova, M. J. de Andrade, D. Kim, H. J. Sim, X. Lepró, A. A. Zakhidov, J.-B. Lee, G. M. Spinks, S. Roth, S. J. Kim, and R. H. Baughman, Adv. Mater. 28, 5038-5044 (2016).

${ }^{14}$ V. Bhatnagar and P. Owende, Energy Sci. Eng. 3, 153 (2015).

${ }^{15}$ M. S. White, M. Kaltenbrunner, E. D. Głowacki, K. Gutnichenko, G. Kettlgruber, I. Graz, S. Aazou, C. Ulbricht, D. A. M. Egbe, M. C. Miron, Z. Major, M. C. Scharber, T. Sekitani, T. Someya, S. Bauer, and N. S. Sariciftci, Nat. Photonics 7, 811-816 (2013).

${ }^{16}$ M. Kaltenbrunner, T. Sekitani, J. Reeder, T. Yokota, K. Kuribara, T. Tokuhara, M. Drack, R. Schwödiauer, I. Graz, S. Bauer-Gogonea, S. Bauer, and T. Someya, Nature 499, 458-463 (2013).

${ }^{17}$ M. Kaltenbrunner, M. S. White, E. D. Głowacki, T. Sekitani, T. Someya, N. S. Sariciftci, and S. Bauer, Nat. Commun. 3, 770 (2012).

${ }^{18}$ L. Rauscher, S. Fujimoto, H. T. Kaibe, and S. Sano, Meas. Sci. Technol. 16, 1054 (2005).

${ }^{19}$ P. Kolodner, Rev. Sci. Instrum. 85, 54901 (2014).

${ }^{20}$ D. Liu, Q. Li, W. Peng, L. Zhu, H. Gao, Q. Meng, and A. J. Jin, Rev. Sci. Instrum. 86, 34703 (2015).

${ }^{21}$ J. D'Angelo, A. Downey, and T. Hogan, Rev. Sci. Instrum. 81, 075107 (2010).

${ }^{22}$ J. P. Carmo, J. Antunes, M. F. Silva, J. F. Ribeiro, L. M. Goncalves, and J. H. Correia, Measurement 44, 2194-2199 (2011).

${ }^{23}$ H. Wang, R. McCarty, J. R. Salvador, A. Yamamoto, and J. König, J. Electron. Mater. 43, 2274-2286 (2014).

${ }^{24}$ A. Montecucco, J. Buckle, J. Siviter, and A. R. Knox, J. Electron. Mater. 42, 1966-1973 (2013).

${ }^{25}$ K. Yazawa and A. Shakouri, J. Appl. Phys. 111, 024509 (2012).

${ }^{26}$ L. S. Fletcher, J. Heat Transfer 110(4b), 1059-1070 (1988).

${ }^{27}$ Q. Wei, M. Mukaida, K. Kirihara, and T. Ishida, ACS Macro Lett. 3, 948-952 (2014).

${ }^{28}$ M. T. Dunham, M. T. Barako, S. LeBlanc, M. Asheghi, B. Chen, and K. E. Goodson, Energy 93, 2006-2017 (2015).

${ }^{29}$ T. L. Bergman and F. P. Incropera, Fundamentals of Heat and Mass Transfer (Wiley, 2011). 